\title{
A Review on Pattern Recognition of Components for the Quality evaluation using Machine Vision
}

\author{
Charles Martin Loyola .I ${ }^{\# 1}$, Gokul .S \\ \#1.Sri Ramakrishna Engineering College, 9629111777 \\ \#2.Sri Ramakrishna Engineering College, 9677480362
}

\section{ABSTRACT}

Automatic inspection of pattern of the components in the industry using computer vision and image processing techniques are capable of accurately measuring the quality parameters and effectively monitor the pattern/shape of the component. There has been significant development in the field of machine vision and is widely accepted by all the industries for quality inspection . The usage of machine vision can replace the manual and traditional method of quality inspection.

This paper provides review of the computer vision techniques and their applications for visual inspection and decision making in the field of quality inspection in the manufacturing industries.

Keywords- Machine vision, Image processing, Quality, Pattern/shape

\section{Corresponding Author:Charles Martin Loyola. I, Gokul. S}

\section{INTRODUCTION}

Quality control and assurance is the major need for all the industries to compete in the dynamic and globalized market place. Manufacturing industries implement Total quality Management tools and quality management cycles to assure quality of the components. It is obligatory for the industries to use precision measurement and inspection systems to accurately and effectively inspect the products for quality. Quality inspection of any industrial component can be classified into two categories of quality characteristics, parameters which are quantifiable and attributes which are subjective in nature. Measurement of quantifiable parameters using traditional measurement instruments can give reproducible results and hence it can be relied on. However this process is time consuming. Visual inspection of subjective attributes is highly dependent on individual's decision, varies from person to person and hence unreliable [1]. Inspection can be for quality check regarding geometry and dimensions, texture and colour, surface accuracy, assembling accuracy and operations and process capability. Moreover $100 \%$ inspection being repetitive is tedious and time-consuming when carried out manually or using mechanical measuring instruments. It also requires training and skill development to accurately comment on quality and make decisions. Sometimes the environment in which measurements are to be taken may not be human friendly. Automatic inspection systems using image processing techniques can overcome the limitations mentioned above and significantly improve production 
quality and reduce cost.Machine vision systems are efficient, reliable, robust, fast in operation and cost-effective. The system hardware and software can be affordable to small or medium scale industries and hence provide a cost effective way of quality control [2]. A typical industrial vision system comprises of illuminating source, image capturing device, image processing software and hardware. Typical steps in computer vision systems include image capturing using digital camera, image acquisition in computer followed by preprocessing and analysis. Preprocessing and analysis comprise of filtering, data reduction/feature extraction, segmentation, object detection and recognition, image understanding and optimization. Out of these techniques optimization techniques are auxiliary techniques that support the other steps. Preprocessing techniques most commonly consist of noise suppression, deblurring, image enhancement and edge detection. Data reduction step mainly comprises of compression and feature extraction.Object recognition i.e. locating the positions and possibly orientations in an image is based on the principle of template matching [3].

\section{LITERATURE REVIEW}

\section{A. EDGE DETECTION ALGORITHMS:}

Edge detection is a fundamental low level processing and is essential for identifying and classifying objects in an image. Two main principles of edge detection are first derivative-based edge detection also referred as gradient based edge detection to detect image edges by computing image gradient values, and second derivative-based edge detection by seeking in the second derivative zero-crossing

Two methods of first order derivative edge detection are: evaluating the gradients generated along two orthogonal directions and utilizing a set of discrete edge templates with different orientations.

In the first method, edge is judged to be present if the gradient exceeds defined threshold value. Some of the examples of these types of operator are Perwitt, Robert, Sobel.

Perwitt's edge gradient detector is based on $3 \mathrm{X} 3$ edge detection with row and column gradients normalized. Roberts operator uses partial differential operator which uses approximation between the two adjacent pixels of the diagonal direction of the gradient amplitude in order to detect the edge. It can properly position the edges however is still susceptible to fluctuations. Both Prewitt and Sobel Operator use the same differential and filtering operations, the only difference is in the template. Sobel doubles the north, south, west, and east pixels of the Prewitt operator and hence is more sensitive to diagonal edge than horizontal and vertical edges.

Second method of utilizing a set of discrete edge templates is to convolute an image with a set of template gradient response arrays and direction of the largest gradient determines the edge angle.

Different approaches using second order derivative of edge detection are Laplacian generation in continuous and discrete domain, Laplacian of Gaussian (LoG) Edge Detection in Continuous and Discrete Domain, Directed Second Order Derivative Generation. LOG Operator finds the optimal filter of edge detection by ratio of the signal to noise of an image. Firstly, a Gaussian function is used to low-pass filter the image and then Laplacian operator is used to high-pass filter based on second order derivativebeing zero for edge detection. 
Canny edge detector is widely accepted as standard edge detection algorithm. The steps in the canny edge detector are Smoothing the image with a two dimensional Gaussian, finding edge strength by taking the gradient of the image in $\mathrm{X}$ and $\mathrm{Y}$ direction, computing direction of the edge using gradient in $\mathrm{x}$ and $\mathrm{y}$ direction, relating edge direction to the direction that can be traced in an image, non-maximal suppression of pixels to edges at all the points where gradient is at its maximum and finally edge thresholding referred to as hysteresis where value of each pixel is checked against high threshold and low threshold value to decide if it is an edge pixel.

Gradient based algorithms are very sensitive to noise whereas Canny's edge detection algorithm performs better than other algorithms in almost all the scenarios because parameters like standard deviation of Gaussian filter and threshold values can be tailored in order to adapt to different environments. Hence though being computationally more expensive Canny's edge detection algorithm is preferred over others [4].

\section{B. IMAGE FILTERING ALGORITHMS}

In processing a digital image, there is a possibility of image distortion due to random variations in intensity, variations in illuminations or poor contrast which needs to be filtered out. Image filtering process reduces noise in order to smoothen out to improve image quality and accuracy. Filtering algorithms are mainly classified into linear and non-linear ones. The simplest filter is the mean filter wherein each pixel in the image is replace with the mean of the pixels surrounding it. Linear filters provide simplest, fastest filtering which replaces each pixel with a linear combination of its neighbors'.

Moving average or box blur, Hann window, Gaussian blur are some linear filtering algorithms commonly used. Most common algorithm for nonlinear smoothing is median filtering. Median filter is a local filter whose output value is the middle element of a sorted array of pixel values from the filter window. In order to save large computational time, image enhancement can be carried out using image histogram which is very helpful in calculating median of the pixels [4].

\section{MORPHOLOGICAL OPERATIONS FOR THINNING ALGORITHMS}

Morphology is a tool for extracting image components which are useful in the representation and description of region shape. Morphological techniques typically probe an image with a small template called structuring element (SE). The SE is small compared to the image and its size.

Zeros and ones in matrix define its shape. The SE is placed at all possible points in the image and it is compared with the corresponding neighborhood of pixels. By marking the locations where SE fits (matches) or hits (mismatches) the image. Thinning can be used to create skeleton of an image to take care of connectivity and shape preservation [5].

\section{INDUSTRIAL APPLICATIONS OF MACHINE VISION IN INSPECTION AND QUALITY CONTROL}

Applications of modern industrial machine vision systems can be broadly categorized in four types of inspections: dimensional quality, surface quality, structural quality, operational quality [2]. 


\section{DIMENSIONAL QUALITY}

Badar et al. [6] have demonstrated the use of image processing technique to measure number of teeth, shaft-hole diameter, outer diameter and root diameter of helical gear and concluded that the technique is very useful for quality assurance by providing online inspection facility.

Fang Li [7] have used image processing techniques using MATLAB to obtain the parameters such as pitch diameter, half of thread angle and pitch of thread.

Gadelmawla [8] introduced a machine vision system where 18 common thread

features could be measured for external screw threads which are also compared with reference values for inspecting threads and making decision.

Leta et al. [9] demonstrated the use of computer vision system to measure straightness, hole diameter, roundness and hole centre positions of a thin rectangular object.

Golkar et al. [10] developed an image processing algorithm to measure length of ceramic tiles to improve ceramic tiles classification in terms of size. The border of a ceramic tile is captured by a camera while the conveyor is moving and the image is then processed and analyzed in order to determine the length. The results are found to be in good agreement with the actual values.

\section{SURFACE QUALITY:}

Shivanna et al. [11] proposed a method to evaluate surface roughness parameters of EDM components using machine vision. Images are captured using Charge Coupled Device (CCD) camera and transferred to computer using frame grabber. The surface roughness values are obtained through Image processing in MATLAB.

Perng et al. [12] described a novel optical thread plug gauge (OTPG) composed of industrial endoscope, CCD camera, and two degree of freedom motion control unit for inspection of internal thread to highlight defects such as scratches, collapses and flaws which cannot be detected using conventional thread gauges. They have concluded that the technique surpasses the capability of mechanical gauge and there is a future scope for thread measurement and improvisation using $3 \mathrm{D}$ reconstruction of thread images.

\section{OPERATIONAL QUALITY:}

S. Selvaraj et al. [13] measured warpage of injection moulded plastic components using image processing. The images are captured by using high resolution digital camera held in a fixture and processed using MATLAB image processing tool to compare the warpage of the component with the standard one.

Dhenge et al. [14] proposed a method for recognizing and sorting nuts and bolts using image processing technique. The image captured through high performance camera is applied to Principal Component Analysis (PCA) to extract features and the result is then given to Artificial Neural Network (ANN) to detect the object accurately. They concluded that hardware with belt conveyer can be developed for online detection and sorting of nuts and bolts. 
Hong et al. [15] developed a technique to precisely measure thread starting point and other parameters using two electro-optical techniques for inspecting small size internal threads. First method is employing the "Optimet"Conoprobe sensor and second is using CCD camera and illumination system to acquire 2D thread image. They concluded that the method reduces time, cost and allows $100 \%$ inspection.

Beyer [16] reported a pilot study of deformation analysis of car bodies in crash test using Real-Time Photogrammetric system wherein CCD cameras are used for capturing the image and car stability is assessed by measuring coordinates of different targets.

Mikołajczyk et al. [17] presented a system consisting of USB camera with VGA resolution combined with software tools required to perform tool wear analysis. The software can identify the tool using image segmentation and assess its geometry. Then it uses a knowledge base to determine tool wear.

Khalili et al. [18] proposed a method to measure crack dimensions in solid materials using machine vision. Two ways for dimension measurement using machine vision are, first based on measurement of length of the image skeleton and second of measuring half of the perimeter of crack image.

\section{IN-PROCESS METROLOGY:}

Savio et al. [19] have presented state-of-art techniques in the field of metrology of freeform shapes wherein they state that measurement of product shapes can be used in inprocess metrology to improve accuracy, reduce machining time and also in reverse engineering(CAD model from a physical part). One of the techniques is stand-still optical systems such as Industrial Photogrammetry or Interferometry.

Diab et al. [20] designed on-line visual inspection system capable of testing geometrical quality characteristics of 2D machined products. They have used webcam camera, telecentric lens, LED and positioner. The image is acquired in VP-EYE Image software, then pre-processed in Matlab and analyzed the product geometry to decide if it conforms to pre-specified tolerances using Neural Network Algorithm. The results are compared with statistical approach using correlation and MSE coefficient.

Ayub et al. [21] developed a system for in-line inspection of roundness error using machine vision. The system consisted of CCD camera, holding device for camshaft and DC motor coupled with rotary encoder, interfaced with computer. The roundness error was determined as the minimum radial separation of two bounding concentric circles based on minimum zone circle (MZC) method.

Alshennawy [22] presented visual inspection system using machine vision to perform online inspection of gear to segregate non-conforming products from the production line based on accuracy of holes.

Rosenberger et al. [23] presented an inline measurement system to control quality of springs. A small control loop is provided for statistical process control. Warning and control limits are defined in order to send the feedback to adjust control drive in case of quality offset.

Adamo et al. [24] proposed an inline PC based visual inspection system for glass surface inspection using an array of CMOS cameras, controllable roller conveyor and image processing system. Defects were detected using Canny edge detection along with parallel processing toolbox in MATLAB to reduce time. 
Schellhorn et al. [25] discussed the challenges of implementing in-process metrology for milling. A sensor has been attached in the hollow taper shank tooling in order capture the image.

Chiou et al. [26] used modern sensors and data acquisition systems for online monitoring of CNC machining process to control surface roughness. It is computed using built in softsensors in camera and further analyzed using front script softsensors. The correlation of mean intensity and surface roughness is determined and then compared with control limits for decision making for quality inspection.

\section{CONCLUSION AND FUTURE}

Review of recent advances in visual inspection using image processing techniques gives an insight into widespread applications of the technique in inspection in industries. It is a promising field that provides an effective and economic alternative to traditional manual inspection and has the capability to facilitate $100 \%$ inspection. The various directions to the work which can be carried out further are faster algorithms to allow real time monitoring, standard datasets and well-defined protocols for fair analysis, generalization of technique for multiple products and defects, applications in industrial production for quality control.

\section{REFERENCES}

[1]Peansupap, V., and C. Laofor. "Digital Image Processing for Evaluating Defect Level in Visual Quality Inspection." W078Special Track 18th CIB World Building Congress May 2010 Salford, United Kingdom.

[2] Malamas, Elias N., et al. "A survey on industrial vision systems, applications and tools." Image and vision computing 21.2 (2003): 171-188.

[3] Egmont-Petersen, Michael, Dick de Ridder, and Heinz Handels. "Image processing with neural networks - a review." Pattern recognition 35.10 (2002): 2279-2301.

[4] Johnny Canny. "A Computational Approach to Edge Detection." IEEE Tractions on pattern analysis and machine intelligence, Vol. Pami-8 No. 6 November 1986

[5] Bin, Li, and Mehdi SamieiYeganeh. "Comparison for image edge detection algorithms."IOSR Journal of Computer Engineering 2.6 (2012).

[6] Badar, Nazim, and Imran Amin. "Verifying practical implementation of QA process of mechanical component by using digital image processing." Journal of Independent Studies and Research 12.1 (2014): 63.

[7] Fang Li, - Thread image processing technology research based on combination of edge detection and sub-pixel positioning\|, Journal of Chemical and Pharmaceutical Research, 2015, 7(3):1275-1279. 
[8] Gadelmawla, E. S. "Development Of Computer Vision Algorithms For Measurement And Inspection Of External Screw Threads", Journal of Engineering Sciences, Assiut University, Vol. 39, No 3, pp.581 -605, May 2011.

[9] Leta, Fabiana R., et al. "Discussing accuracy in an automatic measurement system using computer vision techniques." Proceedings of the 18th International Congress of Mechanical Engineering, Nov. 2005.

[10] Golkar, Ehsan, and Anton SatriaPrabuwono. "Vision Based Length Measuring System for Ceramic Tile Borders." Procedia Technology 11 (2013): 771-777.

[11] Shivanna, D. M., M. B. Kiran, and S. D. Kavitha. "Evaluation of 3D surface roughness parameters of EDM components using vision system."Procedia Materials Science 5 (2014): 2132-2141.

[12] Perng, Der-Baau, Ssu-Han Chen, and Yuan-Shuo Chang. "A novel internal thread defect auto-inspection system." The International Journal of Advanced Manufacturing Technology 47.5-8 (2010): 731743.

[13] S.Selvaraj, P.Venkataramaiah, - Measurement of warpage of injection moulded Plastic components using image processingl, International Journal of Innovative Research in Science, Engineering and Technology, ISSN: 2319-8753, Vol. 2, Issue 12, December 2013.

[14] Dhenge, Amol, A. S. Khobragade, and AmbarishSalodkar. "Mechanical Nut-Bolt Sorting using Principle Component Analysis and Artificial Neural Network.", International Journal of Applied Information Systems (IJAIS) - ISSN : 2249-0868, 2nd National Conference on Innovative Paradigms in Engineering \& Technology (NCIPET 2013).

[15] Hong, En, et al. "Non-contact inspection of internal threads of machined parts." The International Journal of Advanced Manufacturing Technology 62.1-4 (2012): 221-229.

[16] Beyer, Horst A. "Automated dimensional inspection with real-time photogrammetry." ISPRS journal of photogrammetry and remote sensing 50.3 (1995): 20-26.

[17] TadeuszMikołajczyk,AdamKłodowski,AdamMrozinski, -Camerabased Automation System for Tool Measurements and Recognition, -9th International Conference Interdisciplinarity in Engineering, INTER-ENG 2015, 8-9 October 2015, TirguMures, Romaniall, Volume 22, 2016, Pages 1035-1042.

[18] Khalili, K., and M. Vahidnia. "Improving the Accuracy of Crack Length Measurement Using Machine Vision."Procedia Technology 19 (2015): 48-55.

[19] Savio, E., Leonardo De Chiffre, and R. Schmitt. "Metrology of freeform shaped parts." CIRP Annals-Manufacturing Technology 56.2 (2007): 810-835. 
[20] Diab, Safaa L., et al. "Designing a Visual Inspection System for Quality Characteristics Dimensions." Mechanical Engineering Research 1.1 (2011): 12.

[21] Ayub, Muhammad Azmi, Azmi B. Mohamed, and Abdul HalimEsa. "In-line inspection of roundness using machine vision."Procedia Technology 15 (2014): 807-816.

[22] Alshennawy, A. A. "Extract the Geometry of Mechanical Parts by Vision System Using Hough Transform." Extraction 12: 15, International Journal of Control, Automation and Systems, ISSN 2165-8277 (print) ISSN 2165-8285 (online), vol.3 no.2 april 2014

[23] Rosenberger, Maik, et al. "Quality control for springs during the production process based on image processing technologies." Proceedings/IMCIC 10 (2010).

[24] Francesco Adamo and Mario Savino, - A low-cost Inspection system for online defects assessment in satin glass, $\|$ 2009, pp. 1304-1311.

[25] Imaging Sensor System with Wireless Data Transmission for Inprocess Measurements in the Machining Area of Milling Centers. UniversitätsbibliothekIlmenau, 2014.

[26] Chiou, Richard Y., et al. "Experimental Study of High Speed CNC Machining Quality by Noncontact Surface Roughness Monitoring." International Journal of Mechanical Engineering and Robotics Research 4.4 (2015): 282. 